Francis Jordan, Department of Mathematics, University of Louisville, Louisville, KY 40292. e-mail: fejord01@athena.louisville.edu

\title{
QUASICONTINUOUS FUNCTIONS WITH A LITTLE SYMMETRY ARE EXTENDABLE
}

\begin{abstract}
It is shown that if a function $f: \mathbb{R} \rightarrow \mathbb{R}$ is quasicontinuous and has a graph which is bilaterally dense in itself, then $f$ must be extendable to a connectivity function $F: \mathbb{R}^{2} \rightarrow \mathbb{R}$ and the set of discontinuity points of $f$ is $f$-negligible. This improves a result of $\mathrm{H}$. Rosen. A similar result for symmetrically continuous functions follows immediately.
\end{abstract}

\section{Introduction}

In [4] H. Rosen proves the following theorem:

Theorem 1. If $f$ is Darboux, quasicontinuous and has a graph whose closure is bilaterally dense in itself, then $f$ is extendable and the discontinuity points of $f$ form an $f$-negligible set.

Our purpose here is to show that the assumption in the theorem above that $f$ be Darboux is redundant. A corollary of this fact this that symmetrically continuous quasicontinuous functions satisfy the conclusion of Theorem 1.

\section{Terminology}

For a set $S \subseteq \mathbb{R}$ we denote its closure by $\operatorname{cl}(S)$. Given $f \in \mathbb{R}^{\mathbb{R}}$ and $x \in \mathbb{R}$ we define the right cluster set of $f$ at $x$ by

$$
\mathrm{C}^{+}(f, x)=\bigcap_{n=1}^{\infty}(\operatorname{cl}(f[(x, x+1 / n)])) .
$$

Key Words: symmetrically continuous functions, extendable functions, quasicontinuous functions, peripherally continuous functions, Darboux functions

Mathematical Reviews subject classification: Primary 26A15; Secondary 54C30

Received by the editors January 29, 1999 
We define the left cluster set of $f$ at $x$, denoted by $\mathrm{C}^{-}(f, x)$, in a similar way. Finally, we let $\mathrm{C}(f, x)=\mathrm{C}^{-}(f, x) \cap \mathrm{C}^{+}(f, x)$ denote the bilateral cluster set of $f$ at $x$.

We say $f: \mathbb{R} \rightarrow \mathbb{R}$ is a cliquish function provided that for every $x_{0} \in \mathbb{R}$ and $\epsilon>0$ and neighborhood $W$ of $x_{0}$ there is a nonempty open set $W_{0} \subseteq W$ such that $\operatorname{osc}\left(f, W_{0}\right)<\epsilon$. We denote the family of cliquish functions by cliq. It is well known, and easy to prove, that if $f \in$ cliq then the set of continuity points of $f$ is a co-meager subset of $\mathbb{R}$.

We say a function $f$ has closure bilaterally dense in itself provided that for every $x \in \mathbb{R}$ we have $\mathrm{C}^{+}(f, x)=\mathrm{C}^{-}(f, x)$. We will also be concerned with the following families of functions. We give descriptions of these families for general topological spaces, although our discussion will be restricted to the real line. To find out more about the families below see [2], [3], and [1].

Dar: $f \in Y^{X}$ is a Darboux function if and only if $f[C]$ is connected in $Y$ for every connected subset $C$ of $X$.

Con: $f \in Y^{X}$ is a connectivity function if and only if the graph of $f$ restricted to $C$ is connected in $X \times Y$ for every connected subset $C$ of $X$.

Ext: $f \in Y^{X}$ is an extendable function if and only if there is a connectivity function $g: X \times[0,1] \rightarrow Y$ such that $f(x)=g(0, x)$ for every $x \in X$.

Pc: $f \in Y^{X}$ is a peripherally continuous function if and only if for every $x \in X$ and every pair of open sets $U \subset X$ and $V \subset Y$ such that $x \in U$ and $f(x) \in V$ there is an open neighborhood $W$ of $x$ with $\operatorname{cl}(W) \subset U$ and $f[\operatorname{bd}(W)] \subseteq V$, where bd $(W)$ denotes the boundary of $W$.

Qc: $f \in Y^{X}$ is a quasi-continuous function if and only if at each point $p \in X$ the following condition holds: for every open set $U \subseteq X$ with $p \in U$ and open set $V \subseteq Y$ with $f(p) \in V$ there exists a non-empty open set $W \subseteq U$ such that $f[W] \subseteq V$.

It is clear from the definitions that $\mathrm{Qc} \subseteq$ cliq. Given an extendable function $f: \mathbb{R} \rightarrow \mathbb{R}$ we say that $A \subseteq \mathbb{R}$ is $f$-negligible if for any function $g: \mathbb{R} \rightarrow \mathbb{R}$ such that $g=f$ on $\mathbb{R} \backslash A$ and $\left.g\right|_{A} \subseteq \operatorname{cl}(f)$ we have that $g$ is also extendable.

\section{The Results}

Theorem 2. If $f$ is quasi-continuous and has a graph whose closure is bilaterally dense in itself, then $f$ is extendable and the discontinuity points of $f$ form an $f$-negligible set. 
Proof. Notice that quasi-continuity together with the property that the closure of $f$ is bilaterally dense in itself implies that $f$ is peripherally continuous.

By Theorem 1 it is enough for us to show that $f$ is Darboux. Let $[a, b]$ be an interval such that $f(a) \neq f(b)$ and $c$ be strictly between $f(a)$ and $f(b)$. Let $A=\{x \in[a, b]: f(x)>c\}$ and $B=\{x \in[a, b]: f(x)<c\}$. By way of contradiction, assume that $c \notin f[(a, b)]$.

Let $P=\operatorname{cl}(A) \cap \operatorname{cl}(B)$. Suppose there is an isolated point $x \in P$. First notice that $x$ cannot be an endpoint of $[a, b]$. For example, if $x=a$ and $x \in B$, then the only way $x$ could be isolated in $P$ would be if there were an $\epsilon>0$ such that $(a, a+\epsilon) \subseteq A$, but this is impossible by peripheral continuity. Thus, $x$ is not an endpoint of $[a, b]$. Since $x$ is isolated in $P$, there is a $\delta>0$ such that $((x-\delta, x) \subseteq A$ or $(x-\delta, x) \subseteq B)$ and $((x, x+\delta) \subseteq A$ or $(x, x+\delta) \subseteq B)$. Without loss of generality assume that $(x-\delta, x) \subseteq A$. By peripheral continuity, $x \notin B$. Since $x \in \operatorname{cl}(B)$, it follows that $(x, x+\delta) \subseteq B$. Thus, $\mathrm{C}^{-}(f, x) \subseteq[c,+\infty)$ and $\mathrm{C}^{+}(f, x) \subseteq(-\infty, c]$. Since $f$ has closure bilaterally dense in itself, it follows that $\mathrm{C}(f, x)=\{c\}$. In which case, by peripheral continuity, we would have $f(x)=c$. Thus, we may assume that $P$ has no isolated points.

Suppose that $P$ is not nowhere dense. By cliquishness, if $P$ contains a nontrivial interval, then $P$ must contain a continuity point $x$ of $f$. It is easy to see that $f(x)=c$. Thus, we may assume that $P$ is nowhere dense.

The set $\operatorname{cl}(A) \cap \operatorname{cl}(B)$ is nonempty, otherwise $[a, b] \backslash(\operatorname{cl}(A) \cup \operatorname{cl}(B))$ would contain a nonempty open interval upon which $f$ would be constantly equal to $c$, contrary to our assumption.

So, we may assume $P$ is a nonempty, nowhere dense, perfect set. Notice that by quasi-continuity, the sets $A \cap I$ and $B \cap I$ are not contained in $P \cap I$ for any nontrivial open interval $I \subseteq[a, b]$ such that $I \cap P \neq \emptyset$. Let $x_{0} \in P$ be the endpoint of some interval contained in $A$. Since the closure of $f$ is bilaterally dense in itself and $f[A] \subseteq(c,+\infty)$ there is a $\delta_{0}>0$ such that $f\left[\left(x_{0}-\delta_{0}, x_{0}+\right.\right.$ $\left.\left.\delta_{0}\right)\right] \subseteq(c-1,+\infty)$. Since $P$ is perfect, there is an $x_{1} \in\left(x_{0}-\delta_{0}, x_{0}+\delta_{0}\right)$ which is the endpoint of an open interval contained in $B$. Since the closure of $f$ is bilaterally dense in itself and $f[B] \subseteq(-\infty, c)$, there is a $\delta_{1}>0$ such that $f\left[\left(x_{1}-\delta_{1}, x_{1}+\delta_{1}\right)\right] \subseteq(-\infty, c+1 / 2)$ and $\left[x_{1}-\delta_{1}, x_{1}+\delta_{1}\right] \subseteq\left(x_{0}-\delta_{0}, x_{0}+\delta_{0}\right)$. Continue inductively defining $\delta_{n}$ and $x_{n}$ so that the following conditions hold:

(1) $x_{n}$ is an endpoint of an interval contained in $A(B)$ if $n$ is even (odd),

(2) $\left[x_{n}-\delta_{n}, x_{n}+\delta_{n}\right] \subseteq\left(x_{n-1}-\delta_{n-1}, x_{n-1}+\delta_{n-1}\right)$,

(3) if $n$ is even, then $f\left[\left(x_{n}-\delta_{n}, x_{n}+\delta_{n}\right)\right] \subseteq\left(c-1 / 2^{n},+\infty\right)$,

(4) if $n$ is odd, then $f\left[\left(x_{n}-\delta_{n}, x_{n}+\delta_{n}\right)\right] \subseteq\left(-\infty, c+1 / 2^{n}\right)$ and

(5) $\delta_{n}<1 / 2^{n}$. 
Since $P$ is closed and $\lim _{n \rightarrow \infty} \delta_{n}=0$, there is a point $x \in P$ such that $x \subseteq\left[x_{n}-\delta_{n}, x_{n}+\delta_{n}\right]$ for every $n \in \omega$. By (3) and (4) we must have $f(x)=c$. Thus, $f$ is a Darboux function completing the proof.

Since a symmetrically continuous function must have a graph with closure bilaterally dense in itself, we have the following corollary of Theorem 2:

Corollary 3. If $f$ is quasicontinuous and symmetrically continuous, then $f$ is extendable and the discontinuity points of $f$ form an $f$-negligible set.

I do not know if peripheral continuity alone is sufficient to guarantee that a symmetrically continuous function is extendable or even Darboux.

\section{References}

[1] K. Ciesielski, Set Theoretic Real Analysis, J. Appl. Anal., 3(2)(1997), 143-190. (Preprint* available ${ }^{1}$ )

[2] R. G. Gibson and T. Natkaniec, Darboux-like Functions, Real Anal. Exchange, 22(1996-97), 492-533.

[3] T. Natkaniec, Almost Continuity, Real Anal. Exchange, 17(1991-92), $462-520$.

[4] H. Rosen, Darboux Quasicontinuous Functions, Real Anal. Exchange, 23(1997-98), 631-640.

\footnotetext{
${ }^{1}$ Preprints marked by * are available in electronic form and can be accessed from Set Theoretic Analysis Web Page: http://www.math.wvu.edu/homepages/kcies/STA/STA.html.
} 